\title{
A Game with Distorted Information
}

\author{
K. T. Lee \\ Department of Mathematics, University of Malaya, 59100 Kuala Lumpur, \\ Malaysia \\ K. L. Teo \\ Department of Mathematics, University of Western Australia, Nedlands, \\ WA 6009, Australia
}

\begin{abstract}
This article considers a two-person game in which the first player has access to certain information that is valuable but unknown to the second player. The first player can distort the information before it is passed on to the second player. The purpose in distorting the information is to render it as useless as possible to the second player. Based on the distorted information received, the second player then maximizes some given objective. In certain cases he may still be able to use the distorted information, but sometimes the information has been so badly distorted that it becomes completely useless to him. (c) 1993 John Wiley \& Sons, Inc.
\end{abstract}

\section{INTRODUCTION}

Let $\Gamma$ be a two-person zero-sum game whose parameters are described by a quadruple $\left\langle S, p, C,\left\{D_{i j}:(i, j) \in C\right\}\right\rangle$. Here $S$ is a finite set whose elements are ordered pairs $(i, j)$ of positive integers, $p$ is a discrete probability function defined on $S$ by $p_{i j}=\operatorname{prob}\{(i, j)\}, C=\left\{(i, j) \in S: p_{i j}>0\right\}$, and $D_{i j}$ is a nonempty subset of $S$. This quadruple is common knowledge to the two players $P_{1}$ and $P_{2}$. The game $\Gamma$ consists of three sequential moves. In the first move, a chance device uses $p$ to pick a pair $(i, j)$ from $S$. The numbers $i$ and $j$ are then dropped into box 1 and box 2 , respectively. In the second move, $P_{1}$ peeks into the boxes to see the numbers $i$ and $j$. He then closes the boxes, chooses a pair $(k, l)$ from $D_{i j}$, labels $k$ on the lid of box 1 , and labels $l$ on the lid of box 2 . In the third and final move, $P_{2}$ looks at the labels on the lids, selects a box, and receives a payoff equal to the selected box's actual contents. The objective in $\Gamma$ is for $P_{1}$ and $P_{2}$ to minimize and maximize, respectively, this payoff.

Notice the box contents $(i, j)$ are true information, whereas the lid labels $(k$, $l)$ are distorted information. The true information, which is useful to $P_{2}$ to select a correct box, is not accessible to him. Instead, he is given the information which has been distorted by $P_{1}$ to foil his attempt to select a correct box. The amount of distortion $P_{1}$ can introduce is specified by the sets $D_{i j}$. The natural questions to ask here are: How should $P_{1}$ distort the information? Is the distorted infor- 
mation still useful to $P_{2}$, and if the answer is yes, how can he use such distorted information to select a box?

The motivation of $\Gamma$ comes from deception games, first considered by Thompson in his unpublished undergraduate thesis at Harvard University in 1970. Spencer [4] introduced deception games to the open literature when he posed the question of whether the value of a certain deception game is $\frac{1}{2}$. Baston and Bostock [1] settled the existence question by proving very general deception games always have a solution. The formal definition of a deception game can be found in their article [1]. In another related article, Baston and Bostock [2] formulated and solved a cover-up game. The discrete version of this cover-up game may be regarded as a particular case of the game here by taking $S=\{(i$, $j): i, j=1, \ldots, n+1\}, C=\{(i, j): i, j=1, \ldots, n\}, p_{i j}=1 / n^{2}$, and $D_{i j}=$ $\{(i, n+1),(n+1, j)\}$ for each $(i, j)$ in $C$.

\section{THE GAME}

Because $\Gamma$ is a finite game, its value $v$ exists. As it is defined, $\Gamma$ is a game in extensive form. The standard method to solve $\Gamma$ is to convert it to its normal form, that is, to set up the appropriate game matrix. The resulting matrix game can then be solved using linear programming. The main drawback of this method is due to the exponential size of pure strategies involved. To see this is the case, let $|X|$ denote the cardinality of a set $X$. It is easily seen that $P_{1}$ has $\Pi\left|D_{i j}\right|$ pure strategies, where the product is taken over $(i, j) \in C$. Similarly, $P_{2}$ has $2^{|L|}$ pure strategies, where $L=\left\{(k, l): \exists(i, j) \in C\right.$ such that $\left.(k, l) \in D_{i j}\right\}$. For a physical interpretation, $C$ is the set of box contents, whereas $L$ is the set of lid labels. Because of the technical difficulty of solving very large-scale linear programs, we look for a more efficient method to solve $\Gamma$. This leads us to consider behavioral strategies that turn out to offer a computational advantage. Behavioral strategies were first introduced by Kuhn [3] in a seminal article on games in extensive form. A behavioral strategy can be viewed as a local randomization on the occasion of a choice rather than the total randomization of pure strategies made before a play by means of a mixed strategy. We will not elaborate further on behavioral strategies because the method developed here is basically selfcontained.

Consider the linear program $(P)$ :

$$
\max \sum_{(i, j) \in C} p_{i j} y_{i j}
$$

subject to

$$
\begin{aligned}
(j-i) x_{k l}+y_{i j} & \leq j, \quad(i, j) \in C, \quad(k, l) \in D_{i j}, \\
x_{k l} & \leq 1, \\
x_{k l} & \geq 0, \quad(k, l) \in L .
\end{aligned}
$$

The variables in $(P)$ are $x_{k l}$ for $(k, l) \in L$, and $y_{i j}$ for $(i, j) \in C$. An optimal solution exists for $(P)$ because the nonempty feasible set is closed, and the 
objective function is bounded from above. Let $\left\{x_{k l}^{*}, y_{i j}^{*}\right\}$ be an optimal solution, and let $z^{*}$ be the optimal value of $P$. We shall show $v=z^{*}$.

Let $P_{2}$ adopt the strategy $\sigma_{2}$, where he selects boxes 1 and 2 with probabilities $x_{k l}^{*}$ and $1-x_{k l}^{*}$, respectively, if the lid labels are $(k, l)$. We claim that, by using $\sigma_{2}, P_{2}$ can ensure an expected payoff of at least $z^{*}$. Suppose $P_{1}$ sees the box contents $(i, j)$; this even can arise with probability $p_{i j}>0$. If $P_{1}$ changes $(i, j)$ to some $(k, l) \in D_{i j}, P_{2}$ will receive, when using $\sigma_{2}$, an expectation $i x_{k l}^{*}+$ $j\left(1-x_{k l}^{*}\right)$ for this event. Thus $P_{2}$ can ensure an expectation of at least $\min _{(k, l) \in D_{i j}}\left\{i x_{k l}^{*}+j\left(1-x_{k l}^{*}\right)\right\}$ for this event. We claim that

$$
y_{i j}^{*}=\min _{(k, l) \in D_{i j}}\left\{i x_{k l}^{*}+j\left(1-x_{k l}^{*}\right)\right\}, \quad(i, j) \in C .
$$

From (1),

$$
y_{i j}^{*}=\min _{(k, l) \in D_{i j}}\left\{i x_{k l}^{*}+j\left(1-x_{k l}^{*}\right)\right\}, \quad(i, j) \in C .
$$

Suppose the inequality in (3) is strict for some $(i, j) \in C$. Then $y_{i j}^{*}<i x_{k l}^{*}+$ $j\left(1-x_{k l}^{*}\right)$ for each $(k, l) \in D_{i j}$. It is therefore possible to increase this particular $y_{i j}^{*}$ by a small amount, while keeping the values of all other optimal variables in $\left\{x_{k l}^{*}, y_{i j}^{*}\right\}$ unchanged, to obtain a feasible solution to $(P)$ with a larger value of the objective function. This contradiction proves (2). Summing over $(i, j) \in$ $C, P_{2}$ can ensure an expected payoff of at least $\Sigma_{(i, j) \in C} p_{i j} y_{i j}^{*}=z^{*}$. Hence

$$
v \geq z^{*}
$$

To prove the reverse inequality $v \leq z^{*}$, we proceed as follows. For $(i, j) \in$ $C$ and $(k, l) \in L$, define

$$
\delta_{i j}^{k l}= \begin{cases}1, & \text { if }(k, l) \in D_{i j} \\ 0, & \text { otherwise }\end{cases}
$$

The dual of $(P)$ is $(D)$ :

$$
\min \sum_{(k, l) \in L} s_{k l}+\sum_{(i, j) \in C} \sum_{(k, l) \in D_{i j}} j t_{i j}^{k l}
$$

subject to

$$
\begin{aligned}
s_{k l}+\sum_{(i, j) \in C}(j-i) \delta_{i j}^{k l} t_{i j}^{k l} & \geq 0, \quad(k, l) \in L, \\
\sum_{(k, l) \in D_{i j}} t_{i j}^{k l} & =p_{i j}, \quad(i, j) \in C, \\
t_{i j}^{k l} & \geq 0, \quad(i, j) \in C, \quad(k, l) \in D_{i j}, \\
s_{k l} & \geq 0, \quad(k, l) \in L .
\end{aligned}
$$


The variables in $(D)$ are $s_{k l}$ for $(k, l) \in L$, and $t_{i j}^{k l}$ for $(i, j) \in C,(k, l) \in D_{i j}$. Since $(P)$ has an optimal solution, $(D)$ also has an optimal solution $\left\{s_{k l}^{*}, t_{i j}^{k l^{*}}\right\}$ with the optimal value of $z^{*}$.

Let $P_{1}$ adopt the strategy $\sigma_{1}$ where he changes $(i, j)$ to $(k, l) \in D_{i j}$ with probability $t_{i j}^{k l^{*}} / p_{i j}$ if the box contents are $(i, j)$. We claim $P_{1}$ can restrict $P_{2}$ 's expected payoff to at most $z^{*}$. Let $E_{1}(k, l)$ and $E_{2}(k, l)$ denote the contribution to $P_{2}$ 's expected payoff when he chooses box 1 and box 2 , respectively, on seeing $(k, l)$. Then

$$
\begin{aligned}
& E_{1}(k, l)=\sum_{(i, j) \in C} p_{i j} i \delta_{i j}^{k l} t_{i j}^{k l^{*}} / p_{i j}=\sum_{(i, j) \in \mathrm{C}} i \delta_{i j}^{k l} t_{i j}^{k l^{*}}, \\
& E_{2}(k, l)=\sum_{(i, j) \in C} p_{i j} j \delta_{i j}^{k l} t_{i j}^{k l^{*}} / p_{i j}=\sum_{(i, j) \in \mathrm{C}} j \delta_{i j}^{k l} t_{i j}^{k l^{*}} .
\end{aligned}
$$

Notice, for example, by the definition of $E_{1}(k, l)$, we have incorporated the probability of $(k, l)$ occurring into the expression. Now $P_{2}$ can receive, for each $(k, l) \in L$, at most $\max \left\{E_{1}(k, l), E_{2}(k, l)\right\}$, so that he can receive an expected payoff of at most $\Sigma_{(k, l) \in L} \max \left\{E_{1}(k, l), E_{2}(k, l)\right\}$.

We next show this last sum is equal to $z^{*}$. For each $(k, l) \in L$ such that $s_{k l}^{*}>0$, the inequality in (5) must hold as an equality (note our discussion is confined to these constraints at an optimal solution). If not, we can decrease the value of the objective function by reducing the value of this $s_{k l}^{*}$ by a small amount. Hence for $s_{k l}^{*}>0$, we have $E_{1}(k, l)-E_{2}(k, l)=s_{k l}^{*}>0$, so that $E_{1}(k, l)>$ $E_{2}(k, l)$. From (5), for $s_{k l}^{*}=0$, we have $E_{2}(k, l) \geq E_{1}(k, l)$. Let $L_{+}=\{(k, l)$ $\left.\in L: s_{k l}^{*}>0\right\}$ and $L_{0}=\left\{(k, l) \in L: s_{k l}^{*}=0\right\}$. The optimal value of $(D)$ is

$$
\begin{aligned}
z^{*} & =\sum_{(k, l) \in L} s_{k l}^{*}+\sum_{(i, j) \in C} \sum_{(k, l) \in D_{i j}} j t_{i j}^{k l^{*}} \\
& =\sum_{(k, l) \in L} s_{k l}^{*}+\sum_{(i, j) \in C} \sum_{(k, l) \in L} j \delta_{i j}^{k l} t_{i j}^{k l^{*}} \\
& =\sum_{(k, l) \in L}\left\{s_{k l}^{*}+\sum_{(i, j) \in C} j \delta_{i j}^{k l} t_{i j}^{k l^{*}}\right\} \\
& =\sum_{(k, l) \in L}\left\{s_{k l}^{*}+E_{2}(k, l)\right\} \\
& =\sum_{(k, l) \in L_{0}} E_{2}(k, l)+\sum_{(k, l) \in L_{+}}\left\{s_{k l}^{*}+E_{2}(k, l)\right\} \\
& =\sum_{(k, l) \in L_{L, 0}} E_{2}(k, l)+\sum_{(k, l) \in L_{+}} E_{1}(k, l) \\
& =\sum_{(k, l) \in L} \max \left\{E_{1}(k, l), E_{2}(k, l)\right\} .
\end{aligned}
$$


This proves that, by using $\sigma_{1}, P_{1}$ can restrict $P_{2}$ 's expected payoff to at most $z^{*}$. Hence

$$
v \leq z^{*}
$$

From (4) and (6), we have the following theorem.

THEOREM: The value of $\Gamma$ is $v=z^{*}$. The strategies $\sigma_{1}$ and $\sigma_{2}$ are optimal for $P_{1}$ and $P_{2}$, respectively.

We can now address the questions posed earlier. $P_{1}$ should distort the information according to $\sigma_{1}$, while $P_{2}$ should select a box according to $\sigma_{2}$. Next, consider the case when the distorted information is useless to $P_{2}$. Under such a circumstance, he has no choice but to ignore completely the distorted information in his selection of a box. In other words, he does not even have to look at the lid labels. If he always selects box 1 (respectively, box 2), his expected payoff is $\Sigma_{(i, j) \in S} i p_{i j}$ (respectively, $\Sigma_{(i, j) \in S} j p_{i j}$ ). By selecting the appropriate box, $P_{2}$ can obtain an expected payoff equal to the larger of these two quantities, so that

$$
v \geq \max \left\{\sum_{(i, j) \in S} i p_{i j}, \sum_{(i, j) \in S} j p_{i j}\right\} .
$$

If the inequality in (7) holds as an equality, clearly the distorted information is useless to $P_{2}$. On the other hand, if this inequality is strict, then the distorted information is useful to $P_{2}$. This is because, by using it wisely, he can do better than by selecting the box with the larger mean box contents. Thus we have a simple criterion to determine whether the distorted information is useful to $P_{2}$ or not.

\section{A PARTICULAR GAME}

Let $n$ and $r$ be given integers with $n \geq 2$ and $1 \leq r \leq n-1$. Let $N=\{(i$, $j): i, j=1, \ldots, n\}$. Consider the case of $\Gamma$ where $S=C=N, p_{i j}=1 / n^{2}$, $D_{i j}=\{(i, h): h \in\{1, \ldots, n\},|j-h| \leq r\} \cup\{(g, j): g \in\{1, \ldots, n\}$, and $|i-g| \leq r\}$ for $(i, j) \in N$. In other words, the box contents $(i, j)$ are chosen uniformly from $N ; P_{1}$ is allowed to change only one of the box contents to some integer in $\{1, \ldots, n\}$ not more than $r$ units distance away. We denote this game and its value by $\Gamma_{n r}$ and $v_{n r}$, respectively. Note that it is meaningful to define $\Gamma_{n r}$ for $r \geq n$. But since $\Gamma_{n r}=\Gamma_{n, n-1}$ for $r \geq n$, it suffices to consider $r \leq n-$ 1 .

Let $\sigma$ be an optimal strategy of $P_{1}$ in $\Gamma_{n r}$. If $P_{1}$ uses $\sigma$ in $\Gamma_{n, r+1}$, he can restrict $P_{2}$ 's expected payoff to at most $v_{n r}$. This implies

$$
v_{n, n-1} \leq v_{n, n-2} \leq \cdots \leq v_{n 1}
$$

A lower bound on $v_{n r}$ can be easily obtained from (7); that is,

$$
v_{n r} \geq(n+1) / 2, \quad 1 \leq r \leq n-1 .
$$


Our object now is to solve $\Gamma_{n r}$ for various values of $n$ and $r$, subject to $n \geq 2$ and $1 \leq r \leq n-1$. Given fixed $n$ and $r$, we need, in general, to solve either $(P)$ or $(D)$ to obtain a solution to $\Gamma_{n r}$. We will give an explicit solution of $\Gamma_{n r}$ for $n$ arbitrary, and the four cases $r=1,2, n-2, n-1$. Notice these values of $r$ are near the endpoints 1 and $n-1$, these cases being the simplest ones to solve. Computational experience suggests a solution of $\Gamma_{n}$ is quite involved for $r$ near the midpoint of 1 and $n-1$. As before, we denote the box contents by $(i, j)$, and the lid labels by $(k, l)$. To simplify the description of the players' strategies in the proofs of Propositions 1-4 below, we introduce the following convention and notation. When describing a strategy (sometimes called a response) of $P_{1}$, we have to indicate how he changes each $(i, j) \in N$. For brevity, we will only indicate those $(i, j)$ that are actually changed. The remaining $(i, j)$ not explicitly mentioned are assumed to be left unchanged by $P_{1}$. We say $P_{1}$ collapses $B$ (a subset of $N$ ) to mean $P_{1}$ changes each $(i, j) \in B$ with $i \neq j$ to $(i$, $i)$ or $(j, j)$, depending on whether $i<j$ or $i>j$. We denote by $\sigma_{b}$ the strategy of $P_{2}$ where he selects box 1 if $k \geq l$, and box 2 if $k<l$. Basically, $\sigma_{b}$ tells $P_{2}$ to select the box with the bigger lid label. Let $N_{r}=\{(i, j) \in N:|i-j| \leq r\}$.

PROPOSITION 1: $v_{n 1}=a_{n}=\left(4 n^{3}+3 n^{2}-7 n+6\right) / 6 n^{2}, n \geq 2$.

PROOF: Let $P_{2}$ adopt $\sigma_{b}$. It is easy to check that a best response from $P_{1}$ is to change $(i-1, i)$ to $(i, i), i=2, \ldots, n$. Simple calculations show $P_{2}$ will receive an expected payoff of $a_{n}$ against this best response. Since $P_{2}$ can ensure an expected payoff of at least $a_{n}$, we have

$$
v_{n 1} \geq a_{n}
$$

Let $P_{1}$ adopt the strategy where he collapses $N_{1}$. It is again easy to check $P_{1}$ can restrict $P_{2}$ 's expected payoff to at most $a_{n}$, so that

$$
v_{n 1} \leq a_{n}
$$

The result then follows from (10) and (11).

PROPOSITION 2: $v_{n, n-1}=(n+1) / 2, n \geq 2$.

PROOF: Let $P_{1}$ adopt the strategy where he collapses $N$. Using this strategy, $P_{1}$ can restrict $P_{2}$ 's expected payoff to $(n+1) / 2$, so that $v_{n, n-1} \leq(n+1) / 2$. The result then follows from (9). Note that to always select box 1 is an optimal strategy of $P_{2}$.

PROPOSITION 3: Let

$$
b_{n}= \begin{cases}20 / 9, & \text { if } n=3 \\ 21 / 8, & \text { if } n=4 \\ (n+1) / 2, & \text { if } n \geq 5\end{cases}
$$

Then $v_{n \cdot n-2}=b_{n}, n \geq 3$. 
PROOF: The case $n=3$ follows from Proposition 1 .

Next, consider the case $n=4$. Let $P_{2}$ adopt the strategy where he selects box 1 if $(k, l)=(2,3)$; otherwise, he selects the box according to $\sigma_{b}$. A best response from $P_{1}$ is to change $(3,2)$ to $(1,2),(1,2)$ and $(3,4)$ to $(3,2),(1,3)$, and $(2$, $4)$ to $(2,3)$. Simple calculations show $P_{2}$ can ensure an expected payoff of at least $\frac{21}{8}$, so that $v_{42} \geq \frac{21}{8}$. Let $P_{1}$ adopt the strategy where he changes $(1,4)$ and $(3,2)$ to $(1,2),(2,3)$ and $(4,1)$ to $(2,1)$, and collapses $\{(1,2),(1,3),(2,1)$, $(2,4),(3,1),(3,4),(4,2),(4,3)\}$. It is easy to verify $v_{42} \leq \frac{21}{8}$.

Consider now the case $n \geq 5$. Let $P_{1}$ adopt the strategy where he changes

$(1, n)$ to $(1,2)$ with probability $\frac{1}{2}$,

$(n-1,2)$ to $(1,2)$ with probability $\frac{1}{2}$,

$(3,2)$ to $(1,2)$,

$(1, n)$ to $(n-1, n)$ with probability $\frac{1}{2}$.

$(n-1,2)$ to $(n-1, n)$ with probability $\frac{1}{2}$,

$(n-1, n-2)$ to $(n-1, n)$,

$(n, 1)$ to $(2,1)$ with probability $\frac{1}{2}$

$(2, n-1)$ to $(2,1)$ with probability $\frac{1}{2}$,

$(2,3)$ to $(2,1)$,

$(n, 1)$ to $(n, n-1)$ with probability $\frac{1}{2}$,

$(2, n-1)$ to $(n, n-1)$ with probability $\frac{1}{2}$,

$(n-2, n-1)$ to $(n, n-1)$, and

collapses the remaining pairs in $N_{n-2}$.

For each fixed $(k, l) \in\{(1,2),(2,1),(n-1, n),(n, n-1),(1,1), \ldots$, $(n, n)\}, P_{1}$ will receive the same expectation, independent of the box he chooses. This implies $P_{1}$ can restrict $P_{2}$ 's expected payoff to $(n+1) / 2$, so that $v_{n, n-2} \leq$ $(n+1) / 2$. The result then follows from (9).

REMARK: When $n=4$ in Proposition 3, $\sigma_{b}$ is not an optimal strategy of $P_{2}$. If $P_{2}$ adopts $\sigma_{b}$, a best response from $P_{1}$ is to change $(1,3)$ to $(1,1),(2,3)$ to $(2,1),(2,4)$ to $(2,2),(3,2)$ to $(1,2),(1,2)$ and $(3,4)$ to $(3,2),(2,1)$, and $(4,3)$ to $(2,3)$. Thus $P_{2}$ can only ensure an expected payoff of $\frac{5}{2}$, which is less than $\frac{21}{8}$.

PROPOSITION 4: Let

$$
c_{n}= \begin{cases}2, & \text { if } n=3 \\ 21 / 8, & \text { if } n=4 \\ \left(4 n^{3}+3 n^{2}-25 n+36\right) / 6 n^{2}, & \text { if } n \geq 5\end{cases}
$$

Then $v_{n .2}=c_{n}, n \geq 3$.

PROOF: The case $n=3$ follows from Proposition 2, and the case $n=4$ from Proposition 3.

Consider now the case $n \geq 5$. Let $P_{2}$ adopt $\sigma_{b}$. It is easy to check a best response from $P_{1}$ is to change $(1,2)$ to $(3,2),(n, n-1)$ to $(n-2, n-1)$, $(i, i+2)$ to $(i, i),(i+1, i)$ to $(i+1, i+2),(i+1, i+2)$ to $(i+1, i)$, $i=1, \ldots, n-2$. Simple calculations show $P_{2}$ will receive an expected payoff of $c_{n}$ against this best response. Since $P_{2}$ can ensure an expected payoff of at least $c_{n}$, we have $v_{n 2} \geq c_{n}, n \geq 5$. 
It is routine to verify the following strategies of $P_{1}$ can restrict $P_{2}$ 's strategy to at most $c_{n}$, so that $v_{n 2} \leq c_{n}, n \geq 5$. In the strategies below, when we say $P_{1}$ changes $\left(i_{0}, j_{0}\right)$ to $\left(k_{0}, l_{0}\right)$, it is implicitly assumed he also changes $\left(j_{0}, i_{0}\right)$ to $\left(l_{0}\right.$, $\left.k_{0}\right)$.

When $n=5, P_{1}$ changes $(2,1),(2,5),(4,3)$ to $(2,3)$, changes $(1,4),(3,2)$, $(5,4)$ to $(3,4)$, and collapses the remaining pairs in $N_{2}$.

When $n=6, P_{1}$ changes $(2,1)$ with certainty, $(2,5)$ with probability $\frac{1}{2},(4$, $3)$ with probability $\frac{1}{2}$ to $(2,3)$, changes $(3,2),(3,6),(5,4)$ to $(3,4)$, changes $(6,5)$ with certainty, $(2,5)$ with probability $\frac{1}{2},(4,3)$ with probability $\frac{1}{2}$ to $(4,5)$, and collapses the remaining pairs in $N_{2}$.

When $n \geq 7, P_{1}$ changes

(2. 1) to $(2,3),(n, n-1)$ to $(n-2, n-1)$,

$(i, i+3)$ and $(i+2, i+1)$ to $(i, i+1), i=1, \ldots,|n / 2|-1$,

$(i, i+3)$ and $(i+2, i+1)$ to $(i+2, i+3), i=|n / 2|, \ldots, n-3$, and

collapses the remaining pairs in $\mathrm{N}_{2}$. This completes the proof.

Let $n$ be fixed. From (8), as $r$ increases from 1 to $n-1, v_{n r}$ decreases (not necessarily strictly) from $a_{n}$ to $(n+1) / 2$. Let $m$ be the smallest integer such that $v_{n m}=(n+1) / 2 ; m$ exists by Proposition 2 . It follows $v_{n r}=(n+1) / 2$ for $r>m$. The implication here is that, if we solve $\Gamma_{n r}$ in terms of increasing $r$, we can stop as soon as we find the first $r$ with $v_{n r}=(n+1) / 2$. In $\Gamma_{n r}$, the distorted information is useful or not to $P_{2}$, depending whether $r<m$ or $r \geq m$. Given $n$, the value of $m$ can be obtained by solving $\Gamma_{n r}$ for a few values of $r$ chosen iteratively, say, by a bisection method. For example, when $n=5$, we have $m=3$.

\section{CONCLUSIONS}

The primary purpose of this article is to investigate how a person $P_{2}$ can make a good decision based on some distorted information. In particular, we would like to identify the condition under which this information is useful to him. We assume the worst-case scenario - the information has been deliberately distorted by a clever adversary $P_{1}$ to penalize him as much as possible. This assumption may be questionable in some situations. However, if $P_{2}$ finds the information distorted from such an adversary useful, then of course he can be certain of the usefulness of such information distorted by nature or lesser adversaries. We formulate a simple game with distorted information in terms of two boxes in Section 2, and show how to solve it using linear programming. Note that the method there can be easily extended to solve the problem with any finite number of boxes, each box containing one number. We have not analyzed this more general problem due mainly to the complexity in notation. In Section 3, we discuss in greater detail a particular game which is nontrivial to solve explicitly.

\section{REFERENCES}

[1] Baston, V.J., and Bostock, F.A., "Deception Games," International Journal of Game Theory, 17, 129-134 (1988). 
[2] Baston, V.J., and Bostock, F.A., "A Simple Cover-Up Game," American Mathematical Monthly, 95, 850-854 (1988).

[3] Kuhn, H.W., "Extensive Games and the Problem of Information," in Contributions to the Theory of Games, Volume II, Annals of Mathematical Studies No. 28, Princeton University Press, Princeton, NJ, 1953, pp. 193-216.

[4] Spencer, J., "A Deception Game," American Mathematical Monthly, 80, 416-417 (1973).

Manuscript received November 29, 1992

Revised manuscript received April 15, 1993

Accepted April 26, 1993 